\title{
MICROESTRUTURA E PROPRIEDADES MECÂNICAS DE UM AÇO DE ALTA RESISTÊNCIA SUBMETIDO A RESFRIAMENTO RÁPIDO *
}

Leandro Scheffer Cipriani ${ }^{1}$ Odair José dos Santos ${ }^{2}$ Vicente Tadeu Buono ${ }^{3}$

\section{Resumo}

A microestrutura e as propriedades mecânicas de um aço de alta resistência submetido a resfriamento rápido empregando equipamentos (Roller Quench e CLC Continuous On-Line Control), presentes na linha de laminação a quente da Usina de Ipatinga - Usiminas, foram avaliadas nesse estudo. A microestrutura obtida foi composta por bainita e ferrita, com frações desses constituintes variando em função do equipamento utilizado. Devido a essa diferença, os valores de limite de escoamento e de resistência foram maiores no aço submetido a resfriamento via CLC. O resfriamento imposto via Roller Quench propiciou ao aço uma maior tenacidade, medida pela energia absorvida em ensaio de impacto Charpy.

Palavras-chave: Resfriamento Rápido; Microestrutura; Propriedades Mecânicas; Tenacidade.

\section{MICROSTRUCTURE AND MECHANICAL PROPERTIES OF A HIGH ESTRENGTH STEEL SUBMITTED TO FAST COOLING}

\begin{abstract}
The microstructure and mechanical properties of a high strength steel subjected to fast cooling using the Roller Quench and CLC-Continuous on-Line Control equipments, present in the Usiminas - Ipatinga, were evaluated in this study. In both cases, the microstructure obtained was composed of bainite and ferrite, with fractions of these constituents varying according to the equipment used. Due to this difference, the values of mechanical properties were higher in the steel subjected to CLC cooling. On the other hand, the toughness, measured by the energy absorbed in the Charpy impact test, was higher when the cooling was done using Roller Quench.
\end{abstract}

Keywords: Fast Cooling; Microstructure; Mechanical Properties; Toughness.

1 Engenheiro de Produção, Engenheiro de Produção Sênior, Gerencia Técnica das Laminações, USIMINAS, Ipatinga, MG, Brasil.

2 Engenheiro Metalurgista, M. Sc., Dr., Pesquisador Especialista, Gerência Geral de Pesquisa e Desenvolvimento, USIMINAS, Ipatinga, MG, Brasil.

3 Físico, M.Sc., Dr., Professor Titular do Departamento de Engenharia Metalúrgica e de Materiais da UFMG , Belo Horizonte, MG, Brasil. 


\section{INTRODUÇÃO}

Estudos demonstram que o aumento da taxa de resfriamento na decomposição da austenita altera os constituintes formados e, por consequência, as propriedades mecânicas do produto final. Sendo assim, aços resfriados, rapidamente, após a laminação a quente, apresentam, frequentemente, valores de propriedades mecânicas superiores àqueles submetidos ao resfriamento ao ar [1,2]. Por essa constatação, pesquisas relacionadas a aços de alta e ultra-alta resistência mecânica têm sido desenvolvidas considerando os efeitos da taxa de resfriamento após sua laminação, objetivando a combinação de processos que minimizem custos e levem a um aumento global de eficiência [3,4]. Dentro desse contexto, os processos em que são asseguradas altas taxas de resfriamento ganham destaque, citando como o principal exemplo, os processos de têmpera que, nos dias atuais, se subdividem em convencional e direta.

O processo de têmpera direta, de elevada eficiência energética, é uma alternativa ao de têmpera convencional, sendo possível resfriar o aço diretamente após a laminação, sem necessidade de reaustenitização. Além disso, segundo a literatura, ele possibilita melhores combinações de propriedades quando comparado aos processos termomecânicos ou tratamento térmico de têmpera convencional ${ }^{[2-4]}$.

De forma a avaliar os efeitos do resfriamento rápido sobre a microestrutura e propriedades mecânicas de um aço de $25 \mathrm{~mm}$ de espessura, empregaram-se os equipamentos Roller Quench e CLC - Continuous On-line Control, presentes na Linha de Laminação a Quente da Usiminas. Desse modo, pode-se comparar a eficiência desses dois equipamentos sobre propriedades desse aço quando submetido a uma mesma taxa de resfriamento, considerando que quando utilizado o Roller Quench ele foi reaustentizado e para o CLC - Continuous On-Line Control não foi reaustentizado.

\section{MATERIAIS E MÉTODOS}

\subsection{Fabricação e processamento do aço}

O processo de fabricação do aço investigado envolveu todas as etapas comumente empregadas em uma aciaria do tipo LD e seu lingotamento, do tipo contínuo, foi efetuado em máquina tipo vertical-curva. Assim, foram selecionadas duas placas com a faixa de composição química visada, apresentada na tabela 1, para serem laminadas em uma espessura final de $25 \mathrm{~mm}$.

Tabela 1: Faixa de composição química visada para o aço estudado (\% em peso)

\begin{tabular}{c|c|c|c|c|c}
\hline $\mathrm{C}$ & $\mathrm{Mn}$ & $\mathrm{Si}$ & $\mathrm{Al}$ & $\mathrm{V}$ & $\mathrm{Ti}$ \\
\hline $0,12-0,14$ & $1,20-1,30$ & $0,20-0,30$ & $0,020-0,040$ & $0,030-0,040$ & $0,010-0,020$ \\
\hline
\end{tabular}

Das placas laminadas, uma chapa foi submetida ao resfriamento rápido via $C L C$ Continuous On-Line Control e outra via Roller Quench. 


\subsection{Tratamentos térmicos}

A chapa foi resfriada rapidamente após ser laminada com temperatura de acabamento em torno de $900^{\circ} \mathrm{C}$, no equipamento $C L C$-Continuous On-line Control, objetivando uma taxa de $24^{\circ} \mathrm{C} / \mathrm{s}$.

No caso do resfriamento via Roller Quench, a chapa, após ter sido laminada, foi reaquecida até $910^{\circ} \mathrm{C}$ por 1 hora e resfriada uma taxa visada a usada no CLC. A avaliação dessa taxa foi determinada em experimentos anteriores realizados por CRUZ, G.M [5].

\subsection{Ensaios e Análises}

Obtidas as chapas tratadas termicamente, foram avaliadas suas características microestruturais e propriedades mecânicas.

As análises microestruturais consistiram de técnicas metalográficas, utilizando microscopias ótica e eletrônica de varredura. Essas análises foram efetuadas ao longo da espessura das chapas.

As propriedades mecânicas foram avaliadas por ensaios de dureza e tração. Os ensaios de dureza, também executados ao longo da espessura das chapas, seguiram a norma ASTM E92.

Os ensaios de tração foram efetuados em três corpos de prova de cada chapa. Esses corpos de prova possuíam o eixo maior orientado na direção longitudinal de laminação, conforme a norma ASTM A370.

Também foi investigada a tenacidade por ensaio de impacto Charpy empregando variadas temperaturas de ensaio. Os corpos de prova para esse ensaio foram extraídos a $1 \frac{1}{4}$ da espessura das chapas e possuíam dimensão padrão $\left(10 \times 10 \mathrm{~mm}^{2}\right)$ de acordo com a norma ASTM E23.

\section{RESULTADOS E DISCUSSÃO}

\subsection{Microestrutura do aço}

O aspecto típico da microestrutura das chapas após os diferentes processamentos, a $1 / 4$ da espessura, é apresentada na figura 1 . Observou-se a presença de bainita e ferrita em sua constituição. Mesmo não sendo possível medir com precisão a fração desses constituintes nas chapas, pode-se verificar, do ponto de vista qualitativo, uma maior fração de ferrita na chapa submetida ao processamento na Roller Quench. 

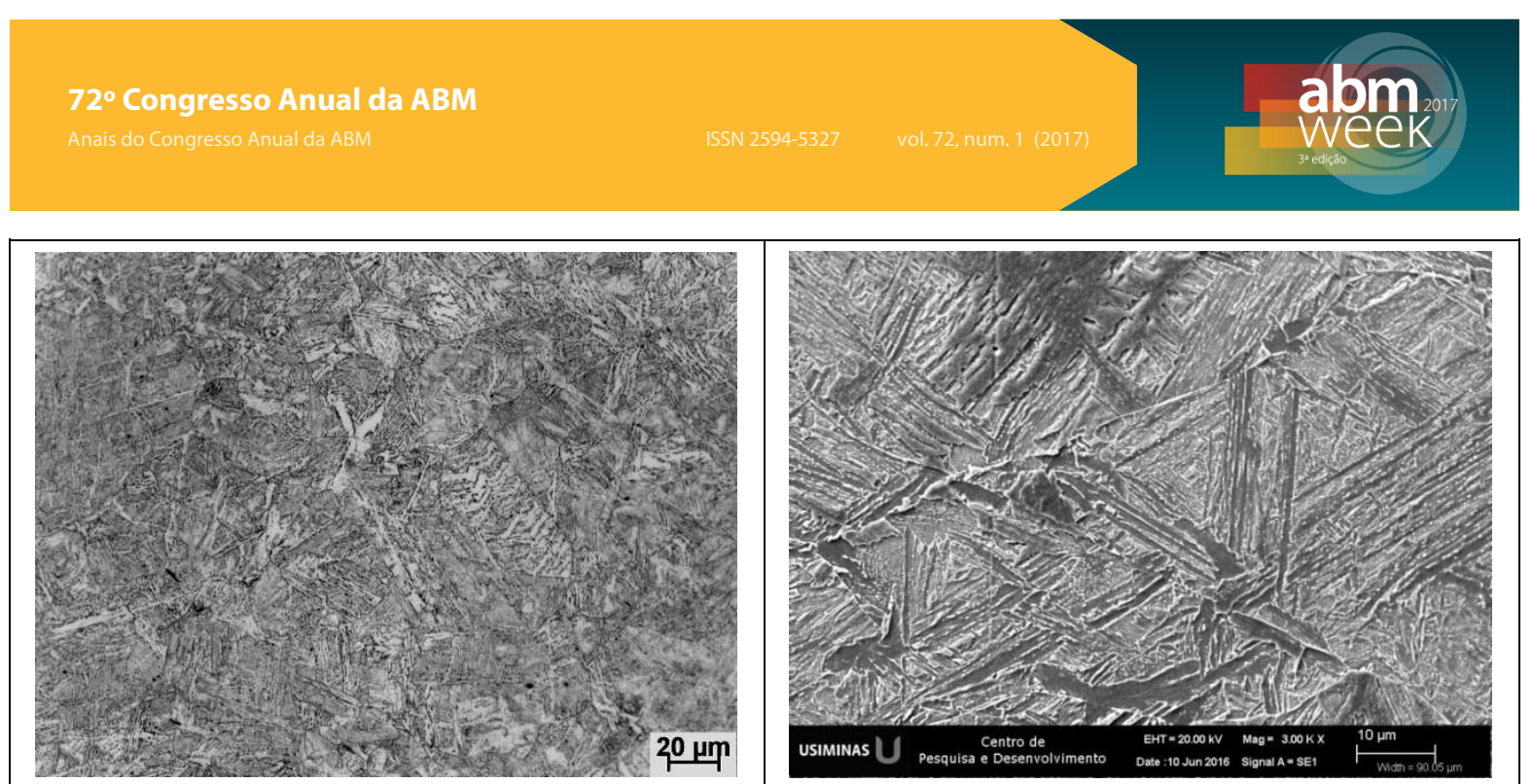

a) resfriamento via CLC
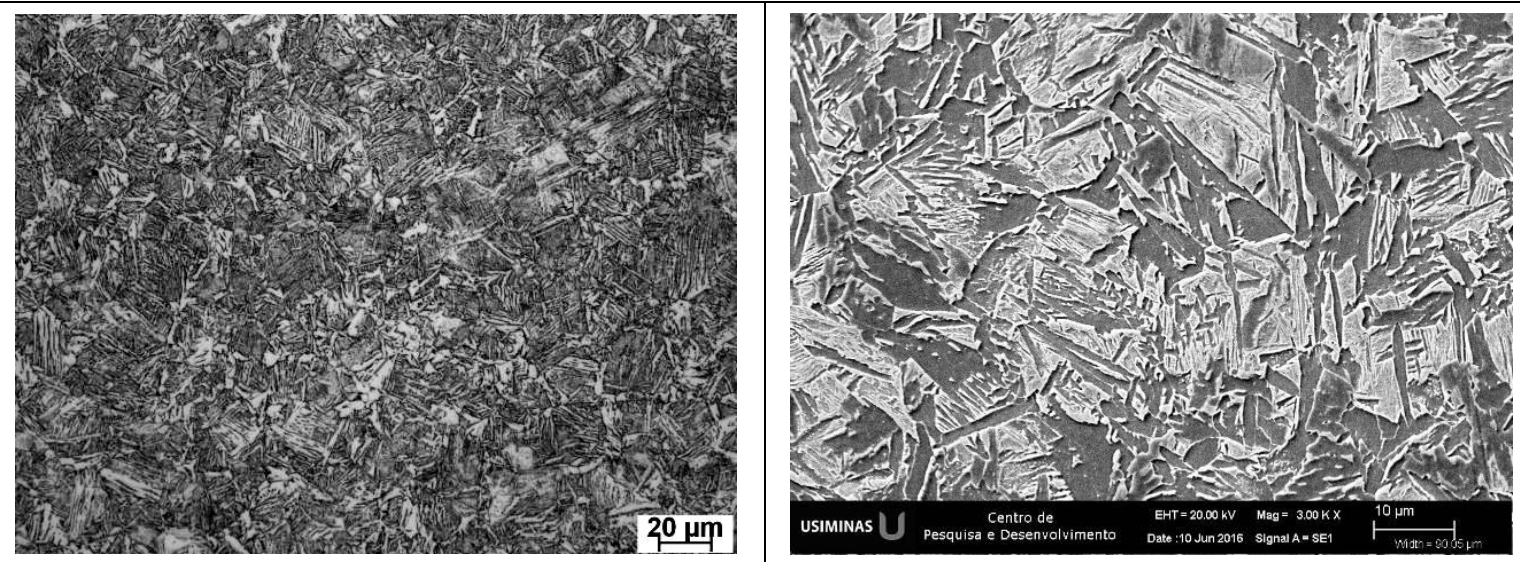

b) resfriamento via Roller Quench

Figura 1. Microestrutura das chapas nas diferentes condições de resfriamento a $1 / 4$ da espessura. Ataque nital 4\%.

Embora tenha sido visado a mesma taxa de resfriamento nos dois processos, as análises microestruturas indicaram uma diferença entre elas. A taxa imposta no resfriamento via Roller Quench deve ter sido menor que a empregada no CLC Continuous On-line Control. Talvez essa diferença possa estar relacionada a diferentes configurações mecânicas desses equipamentos.

\subsection{Propriedades mecânicas}

\subsubsection{Dureza}

Os valores de dureza, medidos ao longo da espessura das chapas, tenderam a exibir um perfil não linear, figura 2. Esse comportamento é devido à maior extração de calor associado às superfícies das chapas, do que em sua região central. Nota-se ainda, nessa figura, que os valores de dureza foram próximos independentemente do processo empregado, apesar das distintas microestruturas obtidas. 


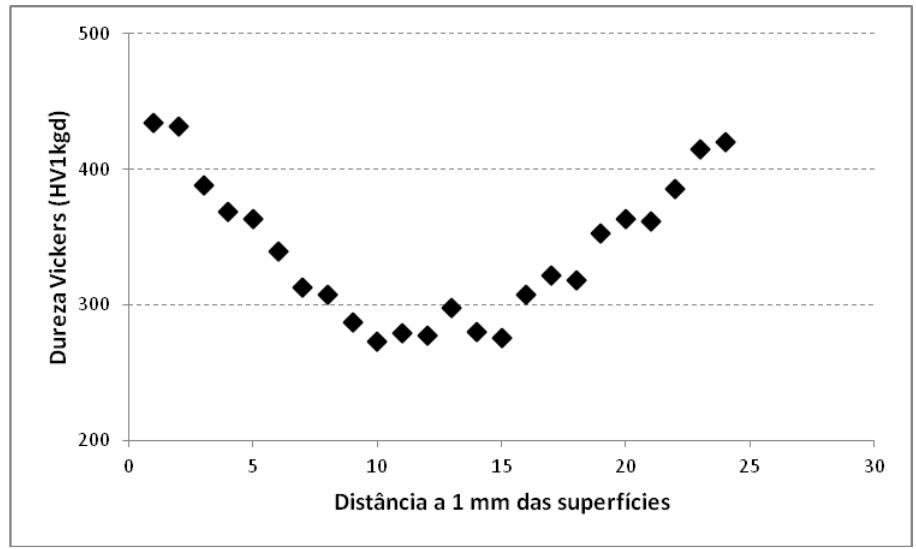

a) Via $C L C$

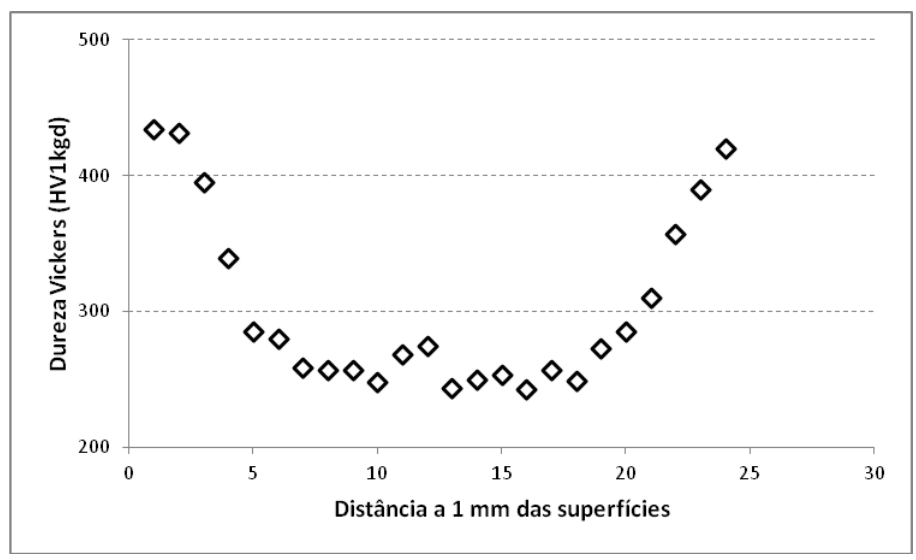

b) Via Roller Quench

Figura 2. Valores de dureza medidos ao longo da espessura das chapas nas diferentes condições de processo.

O valor máximo de dureza, em torno de $440 \mathrm{HV}$, foi obtido nas proximidades das superfícies das chapas. Esse valor é compatível com o obtido em aços completamente martensíticos com mesmo teor de $C$ das chapas avaliadas nesse trabalho, $0,13 \%$ [6]. Isso indica que, possivelmente, nas proximidades das superfícies das chapas houve a formação de uma fina camada de martensita, porém, não observada pelas análises metalográficas realizadas.

\subsubsection{Ensaios de tração}

O limite de escoamento de aços temperados, com estrutura predominantemente martensítica, é função do seu teor de C, equação (1) [6]. Assim, o valor desse parâmetro, considerando o teor de $C$ do aço avaliado e o estimado por essa equação, deveria ser em torno de $1.033 \mathrm{MPa}$. Contudo, isso não foi verificado, como se vê nos resultados exibidos na tabela 2 , onde se encontram os valores médios de limite de escoamento e de resistência dos corpos de prova das chapas.

$$
\sigma_{0,2}(M P a)=413+1,72 \cdot 10^{3}(\% C)^{0,5}
$$


Tabela 2. Limites de escoamento (LE) e de resistência (LR) dos corpos de prova das chapas nas diferentes condições de processamento

\begin{tabular}{ccccc}
\hline \multirow{2}{*}{ Processo } & \multicolumn{2}{c}{ Orientação Longitudinal } & \multicolumn{2}{c}{ Orientação Transversal } \\
\cline { 2 - 5 } & LE (MPa) & LR (MPa) & LE (MPa) & LR (MPa) \\
\hline Roller Quench & $635 \pm 23$ & $781 \pm 36$ & $619 \pm 6$ & $794 \pm 11$ \\
\hline CLC & $767 \pm 53$ & $932 \pm 16$ & $838 \pm 12$ & $957 \pm 17$ \\
\hline
\end{tabular}

Os valores de propriedades mecânicas da chapa submetida ao CLC foram superiores aos obtidos no Roller Quench. Essa diferença foi devido às distintas microestruturas obtidas nesses processos, sendo que a variação na fração de ferrita pode ser suficiente para explicá-la.

A literatura $[2,3,7]$ cita alguns trabalhos em que são apontadas diferenças entre os valores de propriedades mecânicas em tração de aços submetidos a têmpera empregando processos similares aos empregados nesse estudo. Nesses casos, como a microestrutura obtida foi completamente martensítica, essa diferença é justificada pelo condicionamento da austenita anteriormente ao resfriamento. No processo CLC-Continuous On-Line, material é resfriado a partir de condição deformada, logo após a laminação, enquanto no Roller Quench o resfriamento é aplicado após a reaustenitização da chapa a uma temperatura diferente.

\subsubsection{Ensaios de impacto Charpy}

Os valores médios de energia absorvida dos corpos de prova das chapas nas distintas condições de processamento em variadas temperaturas de ensaio são apresentados na tabela 3.

Tabela 3. Valores médios de energia absorvida no ensaio de Charpy pelos corpos de prova das chapas nas diferentes condições de processamento e distintas temperaturas de ensaio.

\begin{tabular}{c|c|c|c|c}
\hline \multirow{2}{*}{ Processo } & \multicolumn{4}{|c}{ Energia absorvida $(\mathrm{J})$} \\
\cline { 2 - 5 } & $-40^{\circ} \mathrm{C}$ & $-20^{\circ} \mathrm{C}$ & $-10^{\circ} \mathrm{C}$ & Ambiente \\
\hline Roller Quench & $95 \pm 6$ & $154 \pm 13$ & $187 \pm 17$ & $228 \pm 13$ \\
\hline CLC & $42 \pm 18$ & $61 \pm 6$ & $79 \pm 3$ & $166 \pm 5$ \\
\hline
\end{tabular}

Do ponto de vista de energia absorvida, o processo Roller Quench demonstrou melhores resultados em relação ao CLC-Continuous On-Line Control. Esse comportamento também é atribuído à maior fração de ferrita presente na microestrutura da chapa processada na Roller Quench. 


\section{CONCLUSÃO}

Tanto no resfriamento via CLC-Continuous On-Line Control quanto no Roller Quench, as chapas apresentaram microestrutura formada por uma mistura de ferrita e bainita, sendo que, qualitatitamente, observou-se uma maior fração de ferrita naquela resfriada via Roller Quench. Isso indicou que embora tenha sido visado a mesma taxa de resfriamento nesses dois processos, a empregada na Roller Quench deve ter sido inferior à do CLC - Continuous On-Line Control.

Pelos resultados desse estudo, além da não necessidade de reaustenitização, podese evidenciar os benefícios do processo CLC - Continuous On-Line Control sobre Roller Quench nas propriedades mecânicas em tração. Essa diferença, por sua vez, foi relacionada à menor fração de ferrita na chapa submetida a essa condição de resfriamento. Essa menor fração de ferrita, por sua vez, resultou em menores valores de energia absorvida em relação ao Roller Quench.

\section{Agradecimentos}

Os autores agradecem à Usiminas, e a CAPES/PROEX.

\section{REFERÊNCIAS}

1 TITHER, G. KEWELL, J. Properties of Directly Quenched and Tempered Structural Steel Plate, Journal of the Iron and Steel Institute. pp. 686-694. Julho/1970.

2 WEISS, R. K.; THOMPSON, S. W. Strength Differences Between Direct-Quenched and Reheated and Quenched Plate. Physical Metallurgy of Direct-Quenched Steels. pp. 107-138. Chicago, 1993.

3 CHANG, W-S. Microstructure and Mechanical Properties of $780 \mathrm{MPa}$ High Strength Steels produced by Direct-quenching and Tempering Process. Journal of Materials Science, vol.37, n.10, pp. 1973-1979. Maio/2002.

4 KESTI, V.; KAIJALAINEN, A.; VÄISÄNEN, A.; JÄRVENPÄÄ, A.; MÄTTÄ; A.; AROLA, A.M., MÄNTYJÄRVI, K.; RUOPPA, R. Bendability and Microstructure of Direct Quenched Optim® 960QC, Materials Science Forum, vols. 783-786, pp. 818-824, 2014.

5 CRUZ, G. M. Avaliação das Variáveis de Processamento de Normalização e Têmpera dos Aços de Classificação ASTM516-70N e USIAR360Q no Forno de Tratamento Térmico 2 da USIMINAS. Dissertação de Mestrado do Programa de Pós-Graduação em Engenharia Metalúrgica e de Minas, Universidade Federal de Minas Gerais, 2003. KRAUSS, G. Martensite in Steel: Strength and Structure. Materials Science and Engineering A, v.272-275, pp. 40-57. 1999.

7 ZHAO, Y.; SHI, J.; CAO, W.; WANG, M.; XIE, G.. Effect of Direct Quenching on Microstructure and Mechanical Properties of Medium-carbon Nb-bearing steel. Journal of Zhejiang University (Applied Physics \& Engineering]. pp. 776-781, 2010.. 